\title{
A Decomposability Property to the Weighted Myerson Value and the Weighted Position Value
}

\author{
Guangming Wang $\mathbb{C}^{1,2}$ and Erfang Shan ${ }^{1}$ \\ ${ }^{1}$ School of Management, Shanghai University, Shanghai 200444, China \\ ${ }^{2}$ School of Mathematical Sciences, University of Jinan, Jinan 250022, China \\ Correspondence should be addressed to Guangming Wang; ss_wanggm@ujn.edu.cn
}

Received 3 April 2020; Accepted 19 January 2021; Published 3 February 2021

Academic Editor: Libor Pekař

Copyright ( 2021 Guangming Wang and Erfang Shan. This is an open access article distributed under the Creative Commons Attribution License, which permits unrestricted use, distribution, and reproduction in any medium, provided the original work is properly cited.

\begin{abstract}
Myerson value and position value are important values in a cooperative game with a graph structure. Usually, due to the differences of players in the game, the weighted Myerson value and weighted position value are more widely used in practical situations. This article offers a new calculation approach of the weighted Myerson value and weighted position value. Instead of using induced games (point game and edge game), we prove that the weighted Myerson value and weighted position value meet decomposability property.
\end{abstract}

\section{Introduction}

Transferable utility game (TU game) [1] usually assumes that a viable alliance can be formed between any players in the game. However, due to the influence of different cultures and backgrounds as well as social classes, hierarchies, organization structure, or communication technology constraints, alliance cannot be formed between any players in the game. In order to solve it, some efforts have been made to deal with this problem. Myerson firstly introduced Myerson value and proposed the method of graph-restricted game (point game), where the points in the graph represent the players in the game, and the edges represent the cooperative relationship between the players [2]. Myerson value is a kind of Shapley value depended on graph [3]. Later, Myerson, Slikker, and van den Nouweland studied other axiomatic characterizations of Myerson value, respectively, see Refs. $[4,5]$. From then on, an increasing interest has been devoted to the study of Myerson value. Li and Shan studied Myerson value on local structures of coalitions and Myerson value for directed graph games, see Refs [6-8]. In addition, van den Nouweland et al. extended the idea initiated by Myerson to hypergraph games and stable system games, respectively, see Refs. $[9,10]$. Motivated by point game, Borm et al. proposed the edge game by using the Shapley value of the edge to obtain the position value [11]. Meessen extended position value on the acyclic graph and axiomatic characterizations of position value [12]. Later, Slikker obtained a complete characterization of the position value on any graph game [13]. Li and Shan studied position value of the structures of graphs [14]. Shan and Zhang investigated position value and the Myerson value for hypergraph [15].

However, in many practical situations, a player in the game has many differences, such as different bargaining powers; it is necessary to give a corresponding weight to each player in the game. Haeringer proposed the weighted Myerson value, that is, the weighted Shapley value of the restricted game [16]. Later, Slikker and van den Nouweland extended the weighted Myerson value to layer structure based on the weight systems of Kalai et al. [17-19]. Xu et al. studied weighted harmonic game and matrix expression of Shapley value [20-23]. To calculate the Myerson value and the position value, we usually need to obtain the corresponding induced game firstly: the point game for the Myerson value and the edge game for the position value. However, induced game is too complex in most cases both in generation and follow-up calculation. Motivated by the above discussion, we investigate an approach that allows us 
to calculate the Myerson value or the position value in a similar way.

The rest of this paper is organized as follows. In Section 2 , we provide some preliminaries on TU games, the weighted graph allocation rule, and the decomposability property. In Section 3, we prove that both weighted Myerson value and weighted position value can be calculated uniformly using the decomposability principle. Section 4 gives conclusions with some remarks.

\section{Preliminaries}

2.1. TU Games. A cooperative game with characteristic function is often referred to as transferable utility game (TU game) [1], represented by a pair $(N, v)$, where $v: 2^{N} \longrightarrow \mathbb{R}$ is a characteristic function with $v(\varnothing)=0$. A value or an allocation rule is a function about the payoff of player in a cooperative game. A very famous allocation rule is the Shapley value:

$$
\operatorname{Sh}_{i}(v)=\sum_{S \subseteq N} \frac{(|N|-|S|) !(|S|-1) !}{|N| !}(v(S)-v(S \backslash\{i\})), \quad i \in N .
$$

2.2. Smallest Connected Set. The triple $(N, v, L)$ represents a graph game, which is composed of two parts: the TU game $(N, v)$ and the graph structure $(N, L)$, $L \subseteq\{\{i, j\} \in N \times N: i \neq j\}$. Let $L_{i}=\{\{i, j\} \in L: j \in N\}$ be the set of edges in the graph $L$ containing point $i \in N$. The dissimilarity points $\left(i_{1}, i_{2}, \ldots, i_{k}\right)$ are called a path of $L$. If there is a path between two points $i, j$, then $i$ and $j$ are connection. If $S$ is connection in $\left(N, L^{S}\right)$ and not connection in $(N, A)$ for any $A \subset L^{S}, A \neq L^{S}$, then we denote by $M C_{L}^{e}(S)$ the smallest connected (edge) set $L^{S} \subset L$ in $(N, L)$.

\subsection{The Weighted Graph Allocation Rule and Decomposability Property}

(a) The weighted graph allocation rule of the point game:

$$
c_{i}^{r^{\theta}}(N, L)=\frac{\theta_{i} \delta_{i}(N, D(L))}{\sum_{j \in D(L)} \theta_{j}}, \quad i \in N,
$$

where $\theta_{i}$ represents the weight of $i$ and $D(L)$ represents the set of nonisolated points in the graph $(N, L)$. In particular, $c_{i}^{r^{\theta}}(N, L)=\left(\left(\delta_{i}(N, D(L))\right) /\right.$ $(|D(L)|))$, if $\theta_{i}=\theta_{j}$ for any $i, j \in N$.

(b) The weighted graph allocation rule of the edge game:

$$
d_{i}^{r^{\theta}}(N, L)=\frac{\sum_{i h \in L_{i}}\left(\theta_{i} /\left(\theta_{i}+\theta_{h}\right)\right)}{|L|}, \quad i \in N
$$

where $\theta_{i}$ represents the weight of $i$ and $D(L)$ represents the set of nonisolated points in the graph $(N, L)$. In particular, $d_{i}^{r^{\theta}}(N, L)=\left(\left(d_{i}(N, L)\right) /\right.$ $(2|L|))$, if $\theta_{i}=\theta_{j}$ for any $i, j \in N$, where $d_{i}(N, L)$ represents the degree of the $i$ in the graph. (c) For any $\left(N, u_{S}, L\right), S \subset N,|S| \geq 2, M C_{L}^{e}(S)=\left\{L_{k}^{S}\right\}_{k=1}^{r(S)}$ $\neq \phi \gamma\left(N, u_{S}, L\right)=\sum_{k=1} r(S) h\left(N, L_{k}^{S}\right)-\sum_{k \neq m} h\left(N, L_{k}^{S}\right.$ $\left.\cup L_{m}^{S}\right)+\cdots+(-1)^{r(S)+1} h\left(N, \cup_{k=1}^{r(S)} L_{k}^{S}\right)$. We will say that $\gamma$ meets decomposability property.

\section{The Decomposability Property of the Weighted Values}

In this section, we calculate the weighted Myerson value and weight position value using the weighted graph allocation rule. It is proved that both weighted Myerson value and weighted position value can be calculated uniformly using the decomposability principle.

Firstly, we introduce the existing approach [11] as follows.

Lemma 1. If $\left(N, u_{S}, L\right) \in C S^{N},|S| \geq 2, M C_{L}^{e}(S)=\left\{L_{k}^{S}\right\}_{k=1}^{r(S)}$, then characteristic function of point game $\left(N, u_{S}^{L}\right)$ is

$$
u_{S}^{L}=\left\{1-\prod_{k=1}^{r(S)}\left[1-u_{D\left(L_{k}^{S}\right)}\right], M C_{L}^{e}(S) \neq \phi, 0, M C_{L}^{e}(S)=\phi .\right.
$$

Lemma 2. If $\left(N, u_{S}, L\right) \in C S_{0}^{N},|S| \geq 2, M C_{L}^{e}(S)=\left\{L_{k}^{S}\right\}_{k=1}^{r(S)}$, then characteristic function of edge game $\left(L, r_{L}^{u_{S}}\right)$ is

$$
r_{L}^{u_{S}}=\left\{1-\prod_{k=1}^{r(S)}\left[1-u_{L_{k}^{S}}\right], M C_{L}^{e}(S) \neq \phi, 0, M C_{L}^{e}(S)=\phi .\right.
$$

Theorem 1. The weighted Myerson value meets the decomposability property.

Proof. If $\quad\left(N, u_{S}, L\right) \in C S^{N},|S| \geq 2, M C_{L}^{e}(S)=\left\{L_{K}^{S}\right\}_{k=1}^{r(S)}$, according to Lemma 1 ,

$$
\begin{aligned}
& \mu^{\theta}\left(N, u_{S}, L\right)= \varphi^{\theta}\left(u_{S}^{L}\right)=\varphi^{\theta}\left(1-\prod_{k=1}^{r(S)}\left[1-u_{\left.\left.D\left(L_{K}^{S}\right)\right]\right),}\right.\right. \\
& 1-\prod_{k=1}^{r(S)}\left[1-u_{\left.D\left(L_{K}^{S}\right)\right]=} \sum_{k=1}^{r(S)} u_{D\left(L_{k}^{S}\right)}-\sum_{k \neq m} u_{D\left(L_{k}^{S}\right) \cup D\left(L_{m}^{S}\right)}\right. \\
&+\cdots+(-1)^{r(S)+1} u_{\cup_{k=1}^{r(S)} D\left(L_{k}^{S}\right)} .
\end{aligned}
$$
have

For any $L_{1}, L_{2} \subset L, D\left(L_{1} \cup L_{2}\right)=D\left(L_{1}\right) \cup D\left(L_{2}\right)$, we

$$
\begin{aligned}
\mu^{\theta}\left(N, u_{S}, L\right)= & \sum_{k=1}^{r(S)} \varphi^{\theta}\left(u_{D\left(L_{k}^{S}\right)}\right)-\sum_{k \neq m} \varphi^{\theta}\left(u_{D\left(L_{K}^{S} \cup L_{m}^{S}\right)}\right) \\
& +\cdots+(-1)^{r(S)+1} \varphi^{\theta}\left(u_{D\left(\cup_{k=1}^{r(S)} L_{k}^{S}\right)}\right) .
\end{aligned}
$$

Using expression in (2), it results in 


$$
\begin{aligned}
\mu^{\theta}\left(N, u_{S}, L\right)= & \sum_{k=1}^{r(S)} c^{r^{\theta}}\left(N, L_{k}^{S}\right)-\sum_{k \neq m} c^{r^{\theta}}\left(N, L_{k}^{S} \cup L_{m}^{S}\right) \\
& +\cdots+(-1)^{r(S)+1} c^{r^{\theta}}\left(N, \cup_{k=1}^{r(S)} L_{k}^{S}\right) .
\end{aligned}
$$

Therefore, the weighted Myerson value meets the decomposability property.

Theorem 2. The weighted position value meets the decomposability property.

Proof. For any $i \in N$,

$$
\begin{aligned}
\pi_{i}^{\theta}\left(N, u_{S}, L\right) & =\sum_{i h \in L_{i}} \frac{\theta_{i}}{\theta_{i}+\theta_{h}} \varphi_{i h}\left(r_{L}^{u_{S}}\right) \\
& =\sum_{j h \in L} \frac{\theta_{j}}{\theta_{j}+\theta_{h}} \delta_{j h}\left(L, L_{i}\right) \varphi_{j h}\left(r_{L}^{u_{S}}\right) .
\end{aligned}
$$

For any $j h \in L, \varphi_{j h}\left(r_{L}^{u_{S}}\right)=\varphi_{j h}\left(1-\prod_{k=1}^{r(S)}\left(1-u_{L_{k}^{S}}\right)\right)$, according to Lemma 2, we have

$$
\begin{aligned}
\varphi_{j h}\left(r_{L}^{u_{S}}\right)= & \sum_{k=1}^{r(S)} \varphi_{j h}\left(u_{L_{k}^{S}}\right)-\sum_{k \neq m} \varphi_{j h}\left(u_{L_{k}^{S} \cup L_{m}^{S}}\right) \\
& +\cdots+(-1)^{r(S)+1} \varphi_{j h}\left(u_{\cup_{k=1}^{r(S)} L_{k}^{S}}\right) .
\end{aligned}
$$

Moreover, we get

$$
\begin{aligned}
\varphi_{j h}\left(r_{L}^{u_{S}}\right)= & \sum_{k=1}^{r(S)} \frac{\delta_{j h}\left(L, L_{k}^{S}\right)}{\left|L_{k}^{S}\right|}-\sum_{k \neq m} \frac{\delta_{j h}\left(L, L_{k}^{S} \cup L_{m}^{S}\right)}{\left|L_{k}^{S} \cup L_{m}^{S}\right|} \\
& +\cdots+(-1)^{r(S)+1} \frac{\delta_{j h}\left(L, \cup_{k=1}^{r(S)} L_{k}^{S}\right)}{\left|\cup_{k=1}^{r(S)} L_{k}^{S}\right|},
\end{aligned}
$$

and then, it results in

$$
\begin{aligned}
& \pi_{i}^{\theta}\left(N, u_{S}, L\right)=\sum_{j h \in L} \frac{\theta_{j}}{\theta_{j}+\theta_{h}} \delta_{j h}\left(L, L_{i}\right) \sum_{k=1}^{r(S)} \frac{\delta_{j h}\left(L, L_{k}^{S}\right)}{\left|L_{k}^{S}\right|}-\sum_{j h \in L} \frac{\theta_{j}}{\theta_{j}+\theta_{h}} \delta_{j h}\left(L, L_{i}\right) \sum_{k \neq m} \frac{\delta_{j h}\left(L, L_{k}^{S} \cup L_{m}^{S}\right)}{\left|L_{k}^{S} \cup L_{m}^{S}\right|} \\
& +\cdots+(-1)^{r(S)+1} \sum_{j h \in L} \frac{\theta_{j}}{\theta_{j}+\theta_{h}} \delta_{j h}\left(L, L_{i}\right) \frac{\delta_{j h}\left(L, \cup_{k=1}^{r(S)} L_{k}^{S}\right)}{\left|\cup_{k=1}^{r(S)} L_{k}^{S}\right|} \\
& =\sum_{j h \in L} \frac{\theta_{j}}{\theta_{j}+\theta_{h}} \sum_{k=1}^{r(S)} \frac{\delta_{j h}\left(L, L_{i} \cap L_{k}^{S}\right)}{\left|L_{k}^{S}\right|}-\sum_{j h \in L} \frac{\theta_{j}}{\theta_{j}+\theta_{h}} \sum_{k \neq m} \frac{\delta_{j h}\left(L, L_{i} \cap\left(L_{k}^{S} \cup L_{m}^{S}\right)\right)}{\left|L_{k}^{S} \cup L_{m}^{S}\right|} \\
& +\cdots+(-1)^{r(S)+1} \sum_{j h \in L} \frac{\theta_{j}}{\theta_{j}+\theta_{h}} \frac{\delta_{j h}\left(L, L_{i} \cap\left(\cup_{k=1}^{r(S)} L_{k}^{S}\right)\right)}{\left|\cup_{k=1}^{r(S)} L_{k}^{S}\right|} \\
& =\sum_{k=1}^{r(S)} \sum_{j h \in L} \frac{\theta_{j}}{\theta_{j}+\theta_{h}} \frac{\delta_{j h}\left(L, L_{i} \cap L_{k}^{S}\right)}{\left|L_{k}^{S}\right|}-\sum_{k \neq m} \sum_{j h \in L} \frac{\theta_{j}}{\theta_{j}+\theta_{h}} \frac{\delta_{j h}\left(L, L_{i} \cap\left(L_{k}^{S} \cup L_{m}^{S}\right)\right)}{\left|L_{k}^{S} \cup L_{m}^{S}\right|} \\
& +\cdots+(-1)^{r(S)+1} \sum_{j h \in L} \frac{\theta_{j}}{\theta_{j}+\theta_{h}} \frac{\delta_{j h}\left(L, L_{i} \cap\left(\cup_{k=1}^{r(S)} L_{k}^{S}\right)\right)}{\left|\cup_{k=1}^{r(S)} L_{k}^{S}\right|} \\
& =\sum_{k=1}^{r(S)} \sum_{i h \in L_{k}^{S}} \frac{\theta_{i}}{\theta_{i}+\theta_{h}} \frac{1}{\left|L_{k}^{S}\right|}-\sum_{k \neq m} \sum_{i h \in L_{k}^{S} \cup L_{m}^{S}} \frac{\theta_{i}}{\theta_{i}+\theta_{h}} \frac{1}{\left|L_{k}^{S} \cup L_{m}^{S}\right|} \\
& +\cdots+(-1)^{r(S)+1} \sum_{i h \in \cup_{k=1}^{r(S)} L_{k}^{S}} \frac{\theta_{i}}{\theta_{i}+\theta_{h}} \frac{1}{\left|\cup_{k=1}^{r(S)} L_{k}^{S}\right|} \\
& =\sum_{k=1}^{r(S)} \frac{\sum_{i h \in L_{k}^{S}}\left(\theta_{i} /\left(\theta_{i}+\theta_{h}\right)\right)}{\left|L_{k}^{S}\right|}-\sum_{k \neq m} \frac{\sum_{i h \in L_{k}^{S} \cup L_{m}^{S}}\left(\theta_{i} /\left(\theta_{i}+\theta_{h}\right)\right)}{\left|L_{k}^{S} \cup L_{m}^{S}\right|}+\cdots+(-1)^{r(S)+1} \frac{\sum_{i h \in \cup_{k=1}^{r(S)} L_{k}^{S}}\left(\theta_{i} /\left(\theta_{i}+\theta_{h}\right)\right)}{\left|\cup_{k=1}^{r(S)} L_{k}^{S}\right|} .
\end{aligned}
$$

Therefore, the weighted position value meets the decomposability property, which completes the proof.
Example 1. Let $\left(N, u_{s}, L\right)$ be a communication situation game with Figure 1, 


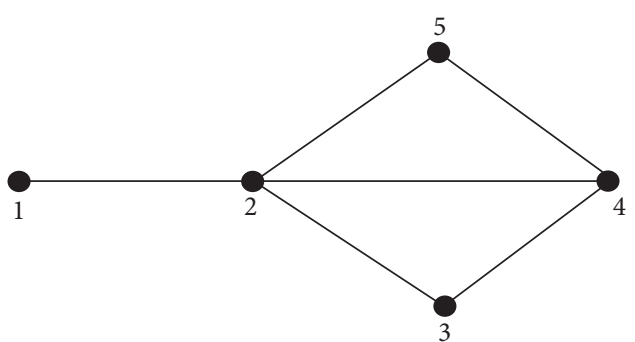

Figure 1: $(N, L)$.

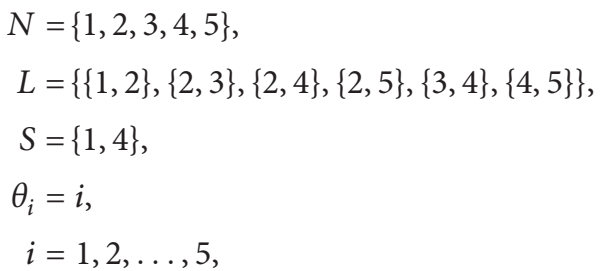

then

$$
\begin{aligned}
M C_{L}^{e} & =\left\{L_{1}^{S}, L_{2}^{S}, L_{3}^{S}\right\}, \\
L_{1}^{S} & =\{\{1,2\}\}\{2,3\},\{3,4\}\}, \\
L_{2}^{S} & =\{\{1,2\},\{2,4\}\}, \\
L_{3}^{S} & =\{\{1,2\},\{2,5\},\{4,5\}\} .
\end{aligned}
$$

$$
\begin{aligned}
\pi^{w}\left(N, u_{\{1,4\}}, L\right)= & \frac{(1 / 3,2 / 3+2 / 5,3 / 5+3 / 7,4 / 7,0)}{3}+\frac{(1 / 3,2 / 3+2 / 6,0,4 / 6,0)}{2} \\
& +\frac{(1 / 3,2 / 3+2 / 7,0,4 / 9,5 / 7+5 / 9)}{3}-\frac{(1 / 3,2 / 3+2 / 5+2 / 6,3 / 5+3 / 7,4 / 6+4 / 7,0)}{4} \\
& -\frac{(1 / 3,2 / 3+2 / 5+2 / 7,3 / 5+3 / 7,4 / 7,5 / 7+5 / 9)}{5} \\
& -\frac{(1 / 3,2 / 3+2 / 6+2 / 7,0,4 / 6+4 / 9,5 / 7+5 / 9)}{4} \\
& +\frac{(1 / 3,2 / 3+2 / 5+2 / 6+2 / 7,3 / 5+3 / 7,4 / 6+4 / 7+4 / 9,5 / 7+5 / 9)}{6} \\
= & \left(\frac{19}{90}, \frac{1613}{3150}, \frac{9}{175}, \frac{17}{105}, \frac{4}{63}\right) .
\end{aligned}
$$

In particular, if $S=\{1,4\}, \theta_{i}=1, i=1,2, \ldots, 5$, the weighted Myerson value and the weighted position value are Myerson value and position value, respectively, the following calculation can be obtained:

$$
\begin{aligned}
\mu\left(N, u_{\{1,4\}}, L\right) & =\left(\frac{1}{3}, \frac{1}{3}, 0, \frac{1}{3}, 0\right), \pi\left(N, u_{\{1,4\}}, L\right) \\
& =\left(\frac{19}{60}, \frac{9}{20}, \frac{1}{20}, \frac{2}{15}, \frac{1}{20}\right) .
\end{aligned}
$$

\section{Conclusion}

In this paper, a simpler weighted graph allocation rule which is used to calculate the weighted values has been investigated. Without the induced games, the weighted Myerson value and weight position value have been calculated by using the weighted graph allocation rule. It has been proved that both the weighted values can be calculated uniformly using the decomposability principle. In addition, it should be pointed out that the obtained results can be 
extended to the future research about Myerson with edge weight and point-edge mixed weight.

\section{Data Availability}

No data were used to support this study. In this paper, the authors only use MATLAB for calculation.

\section{Conflicts of Interest}

The authors declare that they have no conflicts of interest.

\section{References}

[1] R. Branzei, D. Dimitrov, and S. Tijs, Models in Cooperative Game Theory, Springer, Berlin, Germany, 2008.

[2] R. B. Myerson, "Graphs and cooperation in games," Mathematics of Operations Research, vol. 2, no. 3, pp. 225-229, 1977.

[3] L. S. Shapley, "A value for $n$-person games," in Contributions to the Theory of Games II, pp. 307-317, Princeton University Press, Princeton, NJ, USA, 1953.

[4] R. B. Myerson, "Conference structures and fair allocation rules," International Journal of Game Theory, vol. 9, no. 3, pp. 169-182, 1980.

[5] M. Slikker and A. van den Nouweland, Social and Economic Networks in Cooperative Game Theory, Kluwer Academic Publishers, Boston, MA, USA

[6] D. L. Li and E.-F. Shan, "The Myerson value on local structures of coalitions," Journal of the Operations Research Society of China, vol. 7, no. 3, pp. 461-473, 2019.

[7] D. Li and E. Shan, "The Myerson value for directed graph games," Operations Research Letters, vol. 48, pp. 42-46, 2020.

[8] E. Shan, J. Han, and J. Shi, "The efficient proportional Myerson values," Operations Research Letters, vol. 47, no. 6, pp. 574-578, 2019.

[9] A. van den Nouweland, P. Borm, and S. Tijs, "Allocation rules for hypergraph communication situations," International Journal of Game Theory, vol. 20, no. 3, pp. 255-268, 1992.

[10] E. Algaba, J. M. Bilbao, P. Borm, and J. J. López, “The Myerson value for union stable structures," Mathematical Methods of Operations Research, vol. 54, no. 3, pp. 359-371, 2001.

[11] P. Borm, G. Owen, and S. Tijs, "On the position value for communication situations," Siam Journal on Discrete Mathematics, vol. 5, no. 3, pp. 305-320, 1992.

[12] R. Meessen, "Communication games," Master's thesis, Department of Mathematics, University of Nijmegen, Nijmegen, The Netherlands, 1988, in Dutch.

[13] M. Slikker, "A characterization of the position value," International Journal of Game Theory, vol. 33, no. 4, pp. 505-514, 2005.

[14] D. L. Li and E. Shan, "The position value and the structures of graphs," Applied Mathematics and Computation, vol. 356, pp. 190-197, 2019.

[15] E. Shan and G. Zhang, "The position value and the Myerson value for hypergraph communication situations," Static \& Dynamic Game Theory: Foundations \& Applications, pp. 237-250, 2018.

[16] G. Haeringer, "Weighted Myerson value," International Game Theory Review, vol. 2, no. 2, pp. 187-192, 1999.

[17] M. Slikker and A. van den Nouweland, "Communication situations with asymmetric players," Mathematical Methods of Operations Research (ZOR), vol. 52, no. 1, pp. 39-56, 2000.

[18] E. Kalai and D. Samet, "On weighted Shapley values," International Journal of Game Theory, vol. 16, no. 3, pp. 205222, 1987.
[19] A. Ghintran, "Weighted position values," Mathematical Social Sciences, vol. 65, no. 3, pp. 157-163, 2013.

[20] R. Xu and F. Zhang, " $\epsilon$-nash mean-field games for general linear-quadratic systems with applications," Automatica, vol. 114, pp. 108835-108836, 2020.

[21] Y. Wang, T. Liu, and D. Cheng, "From weighted potential game to weighted harmonic game," IET Control Theory \& Applications, vol. 11, no. 13, pp. 2161-2169, 2017.

[22] L. Liu, B. Li, and R. Guo, "Consensus control for networked manipulators with switched parameters and topologies," IEEE Access, vol. 9, p. 9209, 2021.

[23] X. Yi, R. Guo, and Y. Qi, "Stabilization of chaotic systems with both uncertainty and disturbance by the UDE-based control method," IEEE Access, vol. 8, no. 1, pp. 62471-62477, 2020. 